\title{
Visual function in chronic Leber's hereditary optic neuropathy during idebenone treatment initiated 5 to 50 years after onset
}

\author{
Berthold Pemp $^{1}$ (D) $\cdot$ Karl Kircher $^{1} \cdot$ Andreas Reitner $^{1}$
}

Received: 17 June 2019 /Revised: 8 August 2019 / Accepted: 15 August 2019/Published online: 3 September 2019

(C) The Author(s) 2019

\begin{abstract}
Purpose Leber's hereditary optic neuropathy (LHON) is a mitochondrial disease characterized by a subacute and progressive impairment and subsequent degeneration of retinal ganglion cells (RGCs). In most cases, it results in optic nerve atrophy and permanently reduced visual acuity (VA). Idebenone has recently been approved in Europe for treating LHON. However, published clinical data has only focused on efficacy in patients within the first years after disease onset. The present study is the first to evaluate possible effects of idebenone treatment in patients with LHON when initiated after more than 5 years from disease onset.

Methods Oral treatment with idebenone $300 \mathrm{mg}$ tid was started in seven patients 5 to 51 years after LHON onset. All patients had genetically confirmed primary LHON mutations (m11778G $>$ A, m14484T $>$ C, and m13051G $>$ A). Visual function of all fourteen eyes was tested every 3 months using logarithmic reading charts and automated static threshold perimetry. The obtained clinical data were analyzed retrospectively using a multivariate analysis for VA and the Wilcoxon signed-rank test for visual field data.

Results Before treatment, VA was $0.78 \pm 0.38 \log$ MAR (range 0.24 to $1.50 \log$ MAR). During the first year of therapy, VA improved significantly by an average of $-0.20 \pm 0.10 \operatorname{logMAR}$ or $10 \pm 5$ ETDRS letters $(P=0.002$; VA range 0.06 to 1.30 $\operatorname{logMAR})$. Seven of fourteen eyes showed an improvement of 2 or more lines. Visual field mean deviation increased from -8.02 \pm 6.11 to $-6.48 \pm 5.26 \mathrm{~dB}$ after 12 months, but this change was not statistically significant $(P=0.056)$.

Conclusions The increase in VA of patients who have had LHON for more than 5 years observed soon after start of treatment may not constitute a coincidental spontaneous recovery. We hypothesize that the treatment response in chronic LHON was the result of a reactivated signal transduction in surviving dysfunctional RGCs. The results of this study indicate a beneficial effect of idebenone on improvement of visual function in LHON patients with established optic atrophy.
\end{abstract}

Keywords Leber's hereditary optic neuropathy $\cdot$ Idebenone $\cdot$ Optic nerve $\cdot$ Mitochondrial disease $\cdot$ Medical treatment $\cdot$ Vision

\section{Introduction}

Leber's hereditary optic neuropathy (LHON) is one of the more frequent mitochondrial diseases of the optic nerve with an estimated prevalence of 1 in 45,000 people in western countries [1]. The disease is characterized by an acute or subacute impairment of retinal ganglion cells (RGCs) and a subsequent degeneration of RGCs and their

Berthold Pemp

berthold.pemp@medunwien.ac.at

1 Neuro-Ophthalmology Clinic, Department of Ophthalmology and Optometry, Medical University of Vienna, Währinger Gürtel 18-20, 1090 Vienna, Austria nerve fibers. This typically results in a decline in visual acuity (VA), permanent central scotoma, and optic nerve atrophy. Most patients experience a bilateral loss of VA to 20/200 or less during the first year after disease onset; nearly all patients have permanent vision loss and most of them are legally registered as visually impaired $[2,3]$.

More than 100 years after the first descriptions of familial case series of LHON patients by Albrecht von Graefe and Theodor Leber in this journal $[4,5]$, several causative mutations in the mitochondrial DNA were identified and their impact on mitochondrial oxidative phosphorylation (OXPHOS) has been studied intensively over the last decades [6]. The insights gained have enabled a search for targeted pharmaceutical treatments. The short-chain benzoquinone idebenone was investigated in a prospective randomized placebo-controlled trial and in a retrospective cohort study which both compared 
LHON patients treated with idebenone to untreated cohorts $[7$, 8]. The results from both studies and from a subsequent expanded access program supported the beneficial use of idebenone in this hitherto untreatable mitochondrial disease. This led to an approval of idebenone by the European Medicine Agency as the first treatment for LHON, under exceptional circumstances because of the rarity and severity of the disease [9]. However, its therapeutic effects are still under investigation and a matter of debate. A recently published international consensus statement evaluated the available evidence about the clinical and therapeutic management of LHON and recommended that idebenone should be the standard therapy for genetically confirmed LHON in the first year after disease onset [10]. It was also determined that no evidence exists for treatment of patients with a disease duration of more than 5 years. Both clinical studies of idebenone treatment in LHON only focused on efficacy in patients within the first years after disease onset: The RHODOS trial included patients up to 5 years after disease onset with an average disease duration of 2 years [7]. In the retrospective study of Carelli et al., only patients treated within 1 year after onset were included [8]. This was done to avoid the period in which the probability of spontaneous recovery of VA is higher (25 years after onset).

In fact, published evidence for treatment of LHON patients with a disease duration of more than 5 years is scarce. Hence, we aim to provide information concerning this topic and report our results of a study in patients treated with idebenone after long-standing LHON.

\section{Methods}

Seven male patients with genetically confirmed LHON mutations were included in this study. All patients had an onset of disease more than 5 years before treatment was initiated and were prescribed oral idebenone (Raxone, Santhera Pharmaceuticals, Pratteln, Switzerland) at the approved dose of $300 \mathrm{mg}$ tid. In adherence to the tenets of the Declaration of Helsinki, all patients provided signed informed consent for an observational study which was approved by the ethics committee of the Medical University of Vienna. Visual function and retinal morphology were assessed every 3 months during the first year of treatment. Best corrected VA was tested using illuminated logarithmic reading charts (ETDRS charts, Precision Vision, Woodstock, IL, USA). Visual field defects were quantified by measurement of mean deviation (MD) in the central visual field using automated static threshold perimetry (HFAII 30-2 Threshold Test, Carl Zeiss Meditec, Jena, Germany). To account for visual field changes in the region supplied by the temporal optic nerve fibers, the six points nasal to the blind spot in the 30 degree test grid were defined as the central cluster of visual field and central cluster deviation (CCD) was calculated as the average from the numerical total deviation map. Macular ganglion cell layer volume (GCLV) and peripapillary retinal nerve fiber layer thickness (RNFLT) were measured with optical coherence tomography (Spectralis OCT, Heidelberg Engineering, Heidelberg, Germany). Biometric data were analyzed retrospectively. Visual acuity was approximately normally distributed at all measurement points, as assessed by the Shapiro-Wilk test $(P>0.05)$. Changes in VA over time and from baseline were evaluated by a general linear model (GLM) for repeated measurements including patient age, time from LHON onset, and RNFLT of the temporal quadrant as covariates. Visual field data (MD and CCD) were not all normally distributed. Changes in visual field parameters from baseline were evaluated using the Wilcoxon signed-rank test. Pearson's correlation coefficients were calculated to evaluate associations between changes in visual function during treatment and biometric measurements at baseline. A $P$ value of 0.05 was considered the level of significance for all calculations. The statistical analysis was conducted using the software STATISTICA (Release 6.1; StatSoft Inc., Tulsa, OK, USA).

\section{Results}

Patient characteristics including previous disease specific treatments and biometric data of all patients and all eyes are summarized in Table 1.

All patients had bilateral optic disc atrophy more prominently in the temporal sectors and diminished macular GCLV in OCT. Before treatment VA of all eyes was $0.78 \pm 0.38$ logMAR (range 0.24 to $1.50 \log$ MAR). After the first 6 months of therapy, VA improved significantly by a mean of $-0.17 \pm 0.10 \log$ MAR $(P=0.002$, GLM; see Fig. 1$)$. Thereafter, VA was stable in most eyes with a mean VA of $0.60 \pm 0.36 \log$ MAR at the last visit of the first treatment year (range 0.06 to $1.34 \log \mathrm{MAR}$ ). The change in VA over time was statistically significant $(P=0.0009$, GLM). Best VA during the first year was significantly better compared with baseline $(P=0.002$, see Table 1$)$ and seven eyes $(50 \%)$ of four patients showed an increase in VA of two or more lines. Visual field mean deviation (MD) improved by $1.78 \pm$ $2.74 \mathrm{~dB}$ after 12 months compared with baseline (Fig. 2), but this change was not statistically significant $(P=0.056$, the Wilcoxon signed-rank test). The best MD during the first year, however, was significantly better compared with baseline $(P=0.003$, see Table 1$)$. CCD improved by $2.6 \pm 4.0 \mathrm{~dB}$ after 12 months, but this was also not statistically significant $(P=0.32)$. Again, the best individual CCD during the first year was significantly better compared with baseline $(P=$ 0.006 , see Table 1). VA increase and change in MD after 12 months were not statistically associated with age, time since LHON onset, or any biometric measurement obtained 


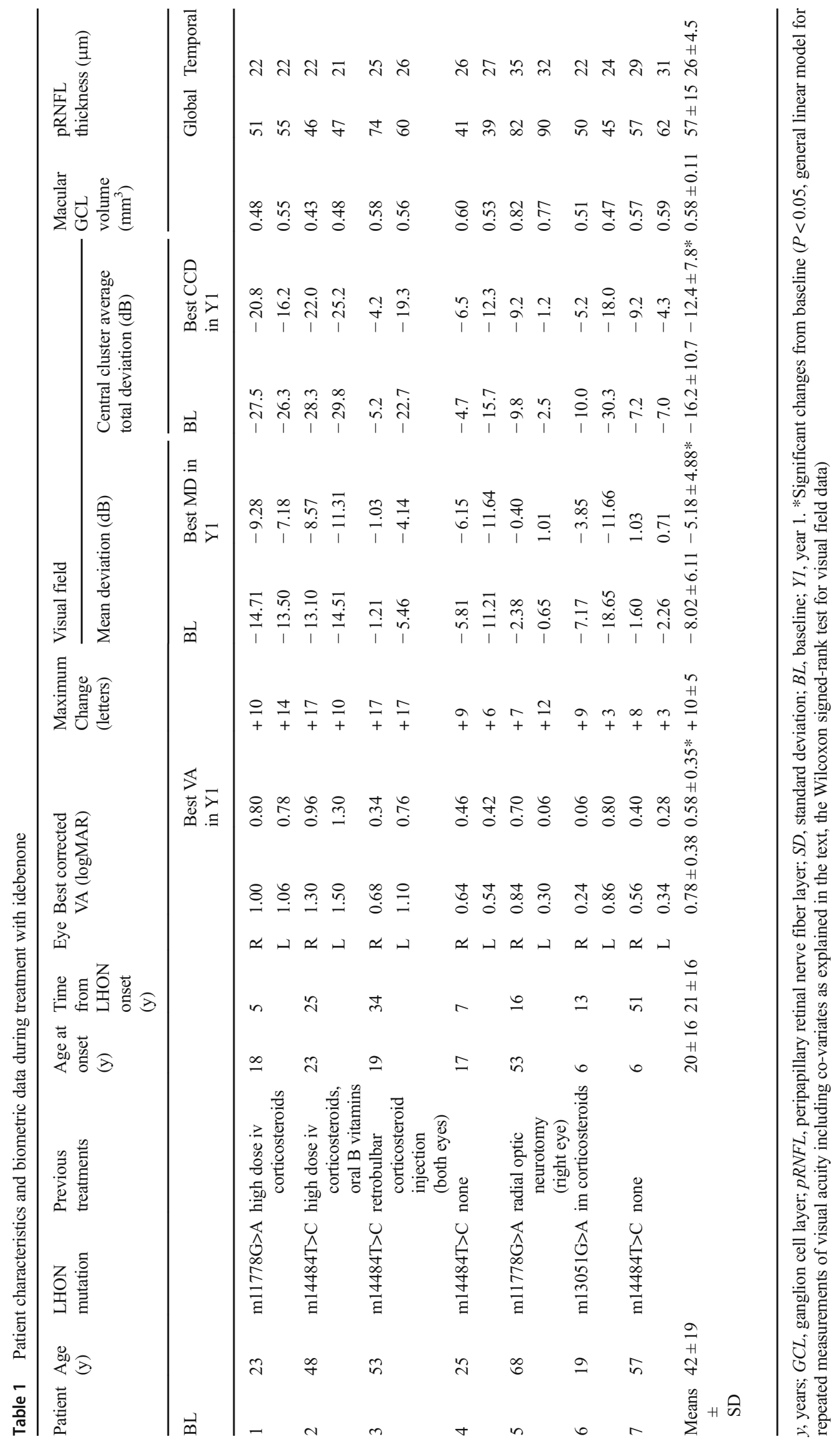




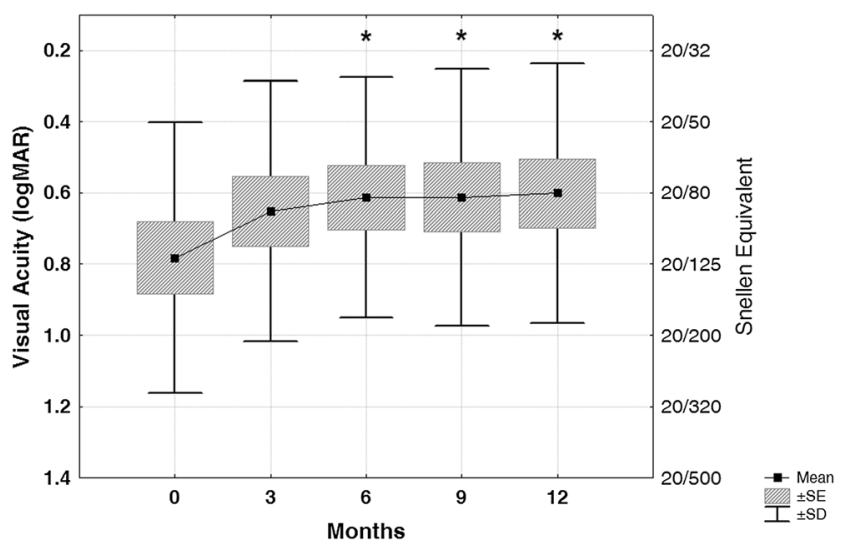

Fig. 1 Visual acuity during treatment with idebenone ( $n=14$ eyes). A significant change over time was detected $(P=0.0009$, general linear model (GLM) for repeated measurements including co-variates as explained in the text). Data are presented as logMAR means \pm standard deviation (SD) and standard error (SE); $\log$ MAR scaling was reversed to depict an increase as an upward shift. * Significant change from baseline $(P<0.012, \mathrm{GLM})$

at baseline. Changes in CCD after 12 months were inversely correlated with baseline $\mathrm{CCD}(r=-0.6, P=0.025)$ and baseline MD $(r=-0.7, P=0.006)$ but were not associated with other baseline data. All OCT parameters remained unchanged during treatment (data not shown).

\section{Discussion}

Our results demonstrate for the first time an improvement of visual function in patients with chronic LHON when treatment with idebenone was initiated later than 5 years and up to 51 years after disease onset. All patients showed an increase in VA of approximately two to three $\log$ MAR lines at least in one eye and this improvement was very concordant during the

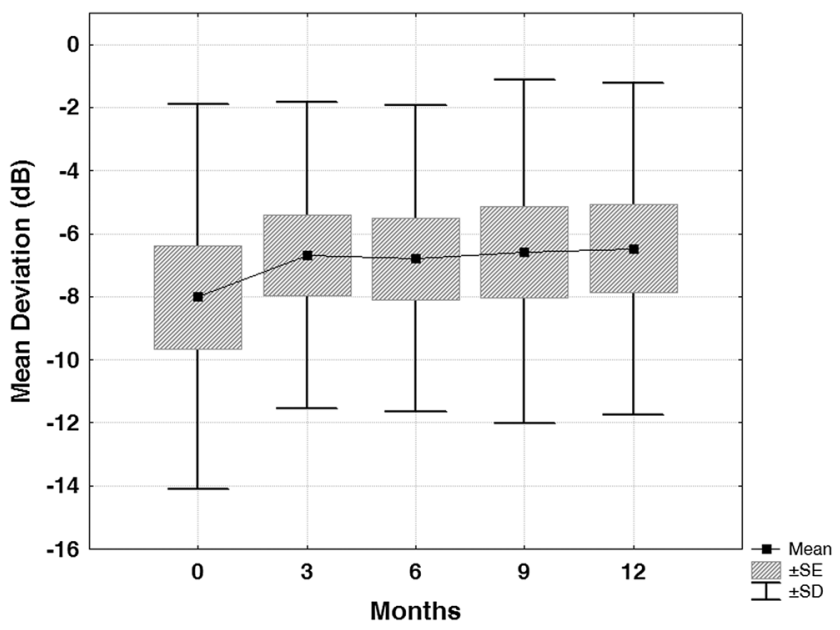

Fig. 2 Visual field mean deviation during treatment with idebenone $(n=$ 14 eyes). Data are presented as means \pm standard deviation (SD) and standard error (SE). Non-significant change from baseline at month 12 $(P=0.056$, the Wilcoxon signed-rank test $)$ first year of treatment. According to the WHO definitions of visual impairment [11], one eye improved from blindness to severe visual impairment, four eyes improved from severe to moderate impairment and three eyes from moderate to mild vision loss. Visual field defects in static perimetry also tended to improve, with the central cluster showing more improvement in visual fields that had more deviation at baseline. Changes in visual function were not dependent on patient age, disease duration or severity of optic atrophy.

We found just one previous case series in the literature, only published in abstract form, that examined 3 chronic patients with a disease duration of 5 or more years under a low dose idebenone treatment of unspecified duration reporting no change in visual function [12].

RHODOS, the only randomized placebo-controlled trial of idebenone in LHON so far, included patients with a mean disease duration of 2 years and nearly all eyes had a VA of $1.0 \log$ MAR or worse at baseline [7]. Averaging results of all eyes, this study showed an increase in VA of less than one line after 3 and 6 months of treatment, which was statistically significant versus placebo. Five treated and 4 untreated patients in this study had a disease duration of 4 to 5 years, but none of them had an improvement of 2 lines or more during the 6 months of observation [9]. Data from the expanded access program, which had longer mean treatment duration but shorter disease duration, showed a mean increase in VA of all included eyes measuring slightly more than 1 line compared with baseline but more than 2 lines compared with the nadir [9]. Due to the progressive loss of visual function reaching a nadir mostly in the first year of LHON, the treatment response compared with baseline values in groups of acute patients may be less pronounced during the first months of treatment and may not be directly comparable to effects in chronic patients in whom no further deterioration is expected.

Idebenone is the first approved treatment for visual loss secondary to LHON, but its benefit compared with the natural history of the disease is still debated, especially because of the possibility of treatment-independent spontaneous recovery during the first few years. Although patients can experience some spontaneous improvement of their visual function mostly in the first months after the nadir, this is less likely to occur years later [13]. However, as single genetically confirmed LHON cases with a substantial spontaneous improvement even after more than 5 years from onset have been reported [13-15], it seems that at least in some patients, an amount of remaining non-apoptotic RGCs enter a dysfunctional dormant state and have the ability to reactivate even after a long time. Our results indicate a beneficial effect of idebenone on such an improvement of visual function in LHON patients with established optic atrophy. The uniform response in our patients with long-standing LHON during the first year after treatment initiation lets us hypothesize that the observed effect may not constitute a coincidental spontaneous recovery but 
was rather treatment-induced. We attribute this to a reactivated signal transduction in surviving dysfunctional RGCs in the chronic disease state. There is growing evidence also from other chronic optic neuropathies, most prominently in glaucoma, that a recovery of visual function can occur following medical treatment, which suggests that it may be possible to restore the function of injured RGC and hence improve vision [16].

The underlying genetics of LHON and its impact on OXPHOS and mitochondrial homeostasis have been intensively studied over the last decades [2, 6, 17]. In most cases, LHON is induced by point mutations in mitochondrial genes encoding for subunits of complex I of the mitochondrial respiratory chain. This may lead to an impaired electron flux in the respiration chain which reduces complex I driven respiration rate and OXPHOS activity and increases the amount of free radicals [18-23]. Alongside augmented reactive oxygen species (ROS) production, increased mitophagy has also been shown in fibroblasts of LHON patients carrying the $\mathrm{m} 13051 \mathrm{G}>\mathrm{A}$ or $\mathrm{m} 11778 \mathrm{G}>\mathrm{A}$ mutations [24]. In patients affected by LHON, mitochondrial mass is reduced compared to unaffected carriers [25], and at some point RGCs seem to lose the ability to compensate for the imbalances in the cellular redox state and for the increased level of free radicals and oxidative damage. These mechanisms may induce a spreading dysfunction of susceptible RGCs in the acute phase of LHON and a subsequent apoptosis of a large amount of such injured cells $[19,22]$. The loss of RGCs in LHON is also accompanied by a demyelination of spared axons in the optic nerve further limiting their capability of conduction [26].

Idebenone is a potent scavenger of free radicals [27] and has also been shown to promote the efficiency of electron transfer to complex III of the mitochondrial respiratory chain [28]. It reduces intracellular ROS and increases ATP production in cells with complex I impairment $[29,30]$. In addition, idebenone has shown to attenuate the aforementioned increase in mitophagy [24] possibly increasing mitochondrial mass. Recent evidence also indicates that a pharmacological reduction of ROS may promote remyelination of surviving denuded axons in models of peripheral, central, and optic nerve injury [31-34]. All these mechanisms could be responsible for restoration of biochemical function in remaining non-apoptotic dysfunctional RGCs and damaged optic nerve axons and could promote signal transduction in the retina and in the optic nerve, making RGCs viable again for transmission of outer retinal signals.

Some limitations must be considered when interpreting our results. Firstly, this is a pilot study in a rare disease lacking a control group. As a single center we were only able to observe a small sample of patients harboring two of the frequent primary LHON mutations and one patient with a rare mutation. Although both patients with the $\mathrm{m} 11778 \mathrm{G}>\mathrm{A}$ mutation showed similar changes in VA, patients with the
m14484T $>C$ or a rare mutation could be more susceptible to improvement [13]. So far, we do not have information about treatment in long-standing LHON patients with the third common primary mutation $\mathrm{m} 3460 \mathrm{G}>\mathrm{A}$. In addition, three of our patients had only little visual field loss and seven of the fourteen eyes had relatively good VA at baseline, which also could have some beneficial effect on the treatment response $[35,36]$. Although a systematic bias due to possible training or placebo effects cannot be completely excluded, we deem this rather unlikely, because the increase in VA exceeded levels of commonly accepted clinical significance in half of the examined eyes. Repeated measurements in the patients of the RHODOS study with longest time from onset did not show a placebo effect of this magnitude. In addition, VA improvement was dissimilar between the two eyes of most patients and was not correlated to baseline VA or other measurements.

The results from the previous clinical studies indicate that the therapeutic potential of idebenone is likely to be the highest if treatment is initiated early in the disease when RGC loss is still low [7, 8]. However, although a growing awareness for rapid diagnosis and therapy of LHON is noted, many patients with chronic disease may not have access to treatment or even be aware of their diagnosis. On the strength of our experience, also patients who have had LHON for a long time can potentially benefit from treatment with idebenone without any severe side-effects. However, the efficacy of idebenone in chronic LHON should be further investigated by larger multi-centered clinical studies.

Acknowledgments Two patients received medication supply by Santhera Pharmaceuticals after approval of the drug was rejected by their responsible health insurance.

Funding Information Open access funding Medical University of Vienna.

\section{Compliance with ethical standards}

Conflict of interest B. Pemp has received travel reimbursements and speaker honoraria from Santhera Pharmaceuticals. K. Kircher and A. Reitner declare that they have no conflict of interest.

Ethical approval All procedures performed in studies involving human participants were in accordance with the ethical standards of the ethics committee of the Medical University of Vienna and with the 1964 Helsinki declaration and its later amendments or comparable standards.

Informed consent Informed consent was obtained from all individual participants included in the study.

Open Access This article is distributed under the terms of the Creative Commons Attribution 4.0 International License (http:// creativecommons.org/licenses/by/4.0/), which permits unrestricted use, distribution, and reproduction in any medium, provided you give appropriate credit to the original author(s) and the source, provide a link to the Creative Commons license, and indicate if changes were made. 


\section{References}

1. Mascialino B, Leinonen M, Meier T (2012) Meta-analysis of the prevalence of Leber hereditary optic neuropathy mtDNA mutations in Europe. Eur J Ophthalmol 22:461-465

2. Yu-Wai-Man P, Griffiths PG, Hudson G, Chinnery PF (2009) Inherited mitochondrial optic neuropathies. J Med Genet 46:145158

3. Kirkman MA, Korsten A, Leonhardt M, Dimitriadis K, De Coo IF, Klopstock T, Griffiths PG, Hudson G, Chinnery PF, Yu-Wai-Man P (2009) Quality of life in patients with Leber hereditary optic neuropathy. Invest Ophthalmol Vis Sci 50:3112-3115

4. von Graefe A (1858) Ein ungewöhnlicher Fall yon hereditiärer Amaurose. Arch Ophthalmol 4:266-268

5. Leber T (1871) Ueber hereditäre und congenital-angelegte Sehnervenleiden. Graefes Arch Ophthalmol 17:249-291

6. Wiggs JL (2015) Mitochondrial genetics and optic neuropathy. Annu Rev Vis Sci 1:97-124

7. Klopstock T, Yu-Wai-Man P, Dimitriadis K, Rouleau J, Heck S, Bailie M, Atawan A, Chattopadhyay S, Schubert M, Garip A, Kernt M, Petraki D, Rummey C, Leinonen M, Metz G, Griffiths PG, Meier T, Chinnery PF (2011) A randomized placebo-controlled trial of idebenone in Leber's hereditary optic neuropathy. Brain 134:2677-2686

8. Carelli V, La Morgia C, Valentino ML, Rizzo G, Carbonelli M, De Negri AM, Sadun F, Carta A, Guerriero S, Simonelli F, Sadun AA, Aggarwal D, Liguori R, Avoni P, Baruzzi A, Zeviani M, Montagna P, Barboni P (2011) Idebenone treatment in Leber's hereditary optic neuropathy. Brain 134:e188

9. European Medicines Agency (2015) Assessment report Raxone. https://www.ema.europa.eu/documents/assessment-report/raxoneepar-public-assessment-report en.pdf. Accessed 12 June 2019

10. Carelli V, Carbonelli M, de Coo IF, Kawasaki A, Klopstock T, Lagrèze WA, La Morgia C, Newman NJ, Orssaud C, Pott JWR, Sadun AA, van Everdingen J, Vignal-Clermont C, Votruba M, YuWai-Man P, Barboni P (2017) International consensus statement on the clinical and therapeutic management of Leber hereditary optic neuropathy. J Neuroophthalmol 37:371-381

11. World Health Organization (2016) International Statistical Classification of Diseases and Related Health Problems 10th Revision, Version 2016. https://icd.who.int/browse10/2016/en\#/ H54.0. Accessed 12 June 2019

12. Carelli V, Ghelli A, Cevoli S, Cortelli P, Lugaresi E, Baruzzi A, Leuzzi V, Degli Esposti M, Barboni P, Montagna P (1998) Idebenone therapy in Leber's hereditary optic neuropathy: report of six cases. Neurology 50(supplement 4):A4

13. Spruijt L, Kolbach DN, de Coo RF, Plomp AS, Bauer NJ, Smeets HJ, de Die-Smulders CE (2006) Influence of mutation type on clinical expression of Leber hereditary optic neuropathy. Am J Ophthalmol 141:676-682

14. Stone EM, Newman NJ, Miller NR, Johns DR, Lott MT, Wallace DC (1992) Visual recovery in patients with Leber's hereditary optic neuropathy and the 11778 mutation. J Clin Neuroophthalmol 12: $10-14$

15. Riordan-Eva P, Sanders MD, Govan GG, Sweeney MG, Da Costa J, Harding AE (1995) The clinical features of Leber's hereditary optic neuropathy defined by the presence of a pathogenic mitochondrial DNA mutation. Brain 118:319-337

16. Fry LE, Fahy E, Chrysostomou V, Hui F, Tang J, van Wijngaarden P, Petrou S, Crowston JG (2018) The coma in glaucoma: retinal ganglion cell dysfunction and recovery. Prog Retin Eye Res 65:7792

17. Maresca A, la Morgia C, Caporali L, Valentino ML, Carelli V (2013) The optic nerve: a "mito-window" on mitochondrial neurodegeneration. Mol Cell Neurosci 55:62-76
18. Howell N (1998) Leber hereditary optic neuropathy: respiratory chain dysfunction and degeneration of the optic nerve. Vis Res 38:1495-1504

19. Brown MD (1999) The enigmatic relationship between mitochondrial dysfunction and Leber's hereditary optic neuropathy. J Neurol Sci 165:1-5

20. Brown MD, Trounce IA, Jun AS, Allen JC, Wallace DC (2000) Functional analysis of lymphoblast and cybrid mitochondria containing the 3460,11778 , or 14484 Leber's hereditary optic neuropathy mitochondrial DNA mutation. J Biol Chem 275:39831-39836

21. Klivenyi P, Karg E, Rozsa C, Horvath R, Komoly S, Nemeth I, Turi S, Vecsei L (2001) Alpha-tocopherol/lipid ratio in blood is decreased in patients with Leber's hereditary optic neuropathy and asymptomatic carriers of the 11778 mtDNA mutation. J Neurol Neurosurg Psychiatry 70:359-362

22. Carelli V, Rugolo M, Sgarbi G, Ghelli A, Zanna C, Baracca A, Lenaz G, Napoli E, Martinuzzi A, Solaini G (2004) Bioenergetics shapes cellular death pathways in Leber's hereditary optic neuropathy: a model of mitochondrial neurodegeneration. Biochim Biophys Acta 1658:172-179

23. Baracca A, Solaini G, Sgarbi G, Lenaz G, Baruzzi A, Schapira AH, Martinuzzi A, Carelli V (2005) Severe impairment of complex Idriven adenosine triphosphate synthesis in leber hereditary optic neuropathy cybrids. Arch Neurol 62:730-736

24. Dombi E, Diot A, Morten K, Carver J, Lodge T, Fratter C, Ng YS, Liao C, Muir R, Blakely EL, Hargreaves I, Al-Dosary M, Sarkar G, Hickman SJ, Downes SM, Jayawant S, Yu-Wai-Man P, Taylor RW, Poulton J (2016) The m.13051G>A mitochondrial DNA mutation results in variable neurology and activated mitophagy. Neurology 86:1921-1923

25. Giordano C, Iommarini L, Giordano L, Maresca A, Pisano A, Valentino ML, Caporali L, Liguori R, Deceglie S, Roberti M, Fanelli F, Fracasso F, Ross-Cisneros FN, D'Adamo P, Hudson G, Pyle A, Yu-Wai-Man P, Chinnery PF, Zeviani M, Salomao SR, Berezovsky A, Belfort R Jr, Ventura DF, Moraes M, Moraes Filho M, Barboni P, Sadun F, De Negri A, Sadun AA, Tancredi A, Mancini M, d'Amati G, Loguercio Polosa P, Cantatore P, Carelli V (2014) Efficient mitochondrial biogenesis drives incomplete penetrance in Leber's hereditary optic neuropathy. Brain 137:335-353

26. Carelli V, Ross-Cisneros FN, Sadun AA (2004) Mitochondrial dysfunction as a cause of optic neuropathies. Prog Retin Eye Res 23: 53-89

27. Mordente A, Martorana GE, Minotti G, Giardina B (1998) Antioxidant properties of 2,3-dimethoxy-5-methyl-6-(10hydroxydecyl)-1,4-benzoquinone (idebenone). Chem Res Toxicol 11:54-63

28. Haefeli RH, Erb M, Gemperli AC, Robay D, Courdier Fruh I, Anklin C, Dallmann R, Gueven N (2011) NQO1-dependent redox cycling of idebenone: effects on cellular redox potential and energy levels. PLoS One 6:e17963

29. Erb M, Hoffmann-Enger B, Deppe H, Soeberdt M, Haefeli RH, Rummey C, Feurer A, Gueven N (2012) Features of Idebenone and related short-chain Quinones that rescue ATP levels under conditions of impaired mitochondrial complex I. PLoS One 7:e36153

30. Yu-Wai-Man P, Soiferman D, Moore DG, Burté F, Saada A (2017) Evaluating the therapeutic potential of idebenone and related quinone analogues in Leber hereditary optic neuropathy. Mitochondrion 36:36-42

31. Caillaud M, Chantemargue B, Richard L, Vignaud L, Favreau F, Faye PA, Vignoles P, Sturtz F, Trouillas P, Vallat JM, Desmoulière A, Billet F (2018) Local low dose curcumin treatment improves functional recovery and remyelination in a rat model of sciatic nerve crush through inhibition of oxidative stress. Neuropharmacology 139:98-116

32. Ghaiad HR, Nooh MM, El-Sawalhi MM, Shaheen AA (2017) Resveratrol promotes remyelination in cuprizone model of multiple 
sclerosis: biochemical and histological study. Mol Neurobiol 54: 3219-3229

33. Naeimi R, Baradaran S, Ashrafpour M, Moghadamnia AA, Ghasemi-Kasman M (2018) Querectin improves myelin repair of optic chiasm in lyolecithin-induced focal demyelination model. Biomed Pharmacother 101:485-493

34. Baradaran S, Hajizadeh Moghaddam A, Ghasemi-Kasman M (2018) Hesperetin reduces myelin damage and ameliorates glial activation in lysolecithin-induced focal demyelination model of rat optic chiasm. Life Sci 207:471-479
35. Nakamura M, Yamamoto M (2000) Variable pattern of visual recovery of Leber's hereditary optic neuropathy. Br J Ophthalmol 84: 534-535

36. Mashima Y, Kigasawa K, Shinoda K, Wakakura M, Oguchi Y (2017) Visual prognosis better in eyes with less severe reduction of visual acuity one year after onset of Leber hereditary optic neuropathy caused by the 11,778 mutation. BMC Ophthalmol 17:192

Publisher's note Springer Nature remains neutral with regard to jurisdictional claims in published maps and institutional affiliations. 